\title{
Boolean Factoring and Decomposition of Logic Networks
}

\author{
Alan Mishchenko Robert Brayton \\ Department of EECS \\ University of California, Berkeley \\ \{alanmi, brayton\}@eecs.berkeley.edu
}

\author{
Satrajit Chatterjee \\ Intel Corporation, Strategic CAD Labs \\ Hillsboro, OR \\ satrajit.chatterjee@intel.com
}

\begin{abstract}
This paper presents new methods for restructuring logic networks based on fast Boolean techniques. The basis for these are 1) a cut-based view of a logic network, 2) exploiting the uniqueness and speed of disjoint-support decompositions, 3) a new heuristic for speeding these up, 4) extending these to general decompositions, and 5) limiting local transformations to functions with 16 or less inputs so that fast truth table manipulations can be used in all operations. Boolean methods lessen the structural bias of algebraic methods, while still allowing for high speed and multiple iterations. Experimental results on K-LUT networks show an average additional reduction of $5.4 \%$ in LUT count, while preserving delay, compared to heavily optimized versions of the same networks.
\end{abstract}

\section{Introduction}

The traditional way of decomposing and factoring logic networks uses algebraic methods. These represent the logic of each node as a sum of products (SOP) and apply algebraic methods to find factors or divisors. Kerneling or two-cube division is used to derive candidate divisors. These methods can be extremely fast if implemented properly, but are biased because they rely on an SOP representation of the logic functions, from which only algebraic divisors are extracted. A long-time goal has been to develop similarly fast methods for finding and using good Boolean divisors, independent of any SOP form.

We present a Boolean method, which uses as its underlying computation, a fast method for disjoint support decomposition (DSD). This approach was influenced by the efficient BDD-based computation of complete maximum DSDs proposed in [6], but we use truth-tables and sacrifice some completeness for speed. However, in practice, we almost always find the maximum DSD. This fast DSD computation is used as the basis for simple nondisjoint decompositions for finding Boolean divisors.

Methods based on these ideas can be seen as a type of Boolean rewriting of logic networks, analogous to rewriting AIG networks [27]. AIG rewriting has been very successful, partly because it can be applied many times due to its extreme speed. Thus, many iterations can be used, spreading the area of change and compensating for the locality of AIG-based transforms. Similar effects can be observed with the new methods.

The paper is organized as follows. Section 2 provides the necessary background on DSD as well as a cut-based view of logic networks. Section 3 shows new results on extending DSD methods to non-disjoint decompositions. A particularly interesting set of applications is on $K$-input lookup-table ( $K$-LUT) networks. Section 4 looks at reducing the number of LUTs after a highquality LUT-mapping and high-effort resynthesis has been done. Implementation details are discussed, followed by experimental results. Section 5 concludes the paper and discusses future applications and improvements.

\section{Background}

A Boolean network is a directed acyclic graph (DAG) with nodes corresponding to logic gates and directed edges corresponding to wires connecting the gates. We use the terms Boolean network, logic network and circuit interchangeably. A $K$-LUT network is a Boolean network whose nodes are $K$-input lookup tables ( $K$-LUTs). A node $n$ has zero or more fanins, i.e. nodes that are driving $n$, and zero or more fanouts, i.e. nodes driven by $n$. The primary inputs (PIs) are nodes of the network without fanins. The primary outputs (POs) are a specified subset of nodes of the network.

An And-Inverter Graph (AIG) is a Boolean network whose nodes are two-input ANDs. Inverters are indicated by a special attribute on the edges of the network.

A cut of node $n$ is a set of nodes, called leaves, such that

1. Each path from any PI to $n$ passes through at least one leaf.

2. For each leaf, there is at least one path from a PI to $n$ passing through the leaf and not through any other leaf.

Node $n$ is called the root of $C$. A trivial cut of node $n$ is the cut $\{n\}$ composed of the node itself. A non-trivial cut is said to cover all the nodes found on the paths from the leaves to the root, including the root but excluding the leaves. A trivial cut does not cover any nodes. A cut is $K$-feasible if the number of its leaves does not exceed $K$. A cut $C_{1}$ is said to be dominated if there is another cut $C_{2}$ of the same node such that $C_{2} \subset C_{1}$.

A cover of an AIG is a subset $R$ of its nodes such that for every $n \in R$, there exists exactly one non-trivial cut $C(n)$ associated with it such that:

1. If $n$ is a PO, then $n \in R$.

2. If $n \in R$, then for all $p \in C(n)$ either $p \in R$ or $p$ is a PI.

3. If $n$ is not a PO, then $n \in R$ implies there exists $p \in R$ such that $n \in C(p)$.

The last requirement ensures that all nodes in $R$ are "used".

We use an AIG accompanied with a cover to represent a logic network. This is motivated by previous work on AIG rewriting and technology mapping. The advantage is that different covers of the AIG (and thus different network structures) can be easily enumerated using fast cut enumeration. The logic function of each node $n \in R$ of a cover is simply the Boolean function of $n$ computed in terms of $C(n)$, the cut leaves. This function can be extracted easily as a truth table using the underlying AIG between the root AIG node and its cut. This computation can be performed 
efficiently as part of the cut computation. For practical reasons, the cuts in this paper are limited to at most 16 inputs ${ }^{1}$.

A completely-specified Boolean function $F$ essentially depends on a variable if there exists an input combination such that the value of the function changes when the variable is toggled. The support of $F$ is the set of all variables on which function $F$ essentially depends. The supports of two functions are disjoint if they do not contain common variables. A set of functions is disjoint if their supports are pair-wise disjoint.

A decomposition of a completely specified Boolean function is a Boolean network with one PO that is functionally equivalent to the function. A disjoint-support decomposition (DSD - also called simple disjunctive decomposition) is a decomposition in which the set of nodes of the resulting Boolean network have disjoint supports. Because of this, the DSD is always a tree (each node has one fanout, including the leaf nodes). The set of leaf variables of any sub-tree of the DSD is called a bound set, the remaining variables are its associated free set. A single disjoint decomposition of a function consists of one block with a bound set as inputs and a single output feeding another block with the remaining (free) variables as additional inputs. A maximal DSD is one where each node cannot be decomposed further by DSD.

It is known that internal nodes of a maximal DSD network can be of only three types: AND, XOR, and PRIME. The AND and XOR nodes may have any number of inputs, while PRIME nodes have at least three inputs and only a trivial DSD. For example, a 2:1 MUX is a prime node with three inputs.

Theorem 2.1 [4]. For a completely specified Boolean function, there is a unique maximal DSD, up to complementation of inputs and outputs of the nodes.

There are several algorithms for computing the maximal DSD [6][39][23]. Our implementation follows [6] but uses truth tables instead of BDDs to manipulate Boolean functions.

\section{General non-disjoint decompositions}

A general decomposition has the form

$$
F(x)=\hat{H}\left(g_{1}(a, b), \cdots, g_{k}(a, b), b, c\right) .
$$

If $|b|=0$ it is called disjoint or disjunctive and if $k=1$ it is called simple.

Definition: A function $F$ has an $(a, b)$-decomposition if it can be written as $F(x)=H(D(a, b), b, c)$ where $(a, b, c)$ is a partition of the variables $x$ and $D$ is a single-output function. $D$ is called the divisor and $H$ the dividend.

Thus an $(a, b)$-decomposition is a simple, non-disjoint decomposition. For general decompositions, there is an elegant theory [36] on their existence. Kravets and Sakallah [19] applied this to constructive decomposition using support-reducing decompositions along with a pre-computed library of gates. In our case, if $|a|>1$, an $(a, b)$-decomposition is support reducing. Although less general, the advantage of $(a, b)$-decompositions is that their existence can be tested very efficiently by using cofactoring and the fast DSD algorithms of this paper. Recent works use ROBDDs to test the existence of decompositions, with the variables $(a, b)$ ordered at the top of the BDD (e.g. see [38] for an easy-to-read description).

The variables $a$ are said to be in a separate block and form the bound set, the variables $c$ are the free set, and the variables $b$ are the shared variables. If $|b|=0$, the decomposition is a DSD.

If a particular cofactor of $F$ with respect to $b$ is independent of $a, a$ is also considered to be in a separate block of the DSD of this

\footnotetext{
1 The fast methods of this paper are based on bit-level truth table manipulations and 16 is a reasonable limit for achieving speed for this.
}

cofactor. We call such cofactors, bound set independent cofactors, or $b s i$-cofactors; otherwise $b s d$-cofactors.

Example: If $F=a b+\bar{b} c$, then $F_{\bar{b}}=c$ is independent of $a$ i.e. it is a $b s i$-cofactor.

A BDD version of the following theorem can be found in [38] as Proposition 2 with $t=1$.

Theorem 3.1: A function $F(a, b, c)$ has an $(a, b)$-decomposition if and only if each of the $2^{|b|}$ cofactors of $F$ with respect $b$ has a DSD structure in which the variables $a$ are in a separate block.

Proof: If. Suppose $F$ has an $(a, b)$-decomposition; then $F(x)=H(D(a, b), b, c)$. Consider a cofactor $F_{b^{j}}(x)$ with respect to a minterm of $b^{j}$, say $b^{j}=b_{1} \bar{b}_{2} \bar{b}_{3} b_{4}$ for $k=4$. This sets $b=1,0,0,1$, giving rise to the function,

$$
F_{b^{j}}(a, c)=H(D(a, 1,0,0,1), 1,0,0,1, c) \equiv H_{b^{j}}\left(D_{b^{j}}(a), c\right) .
$$

Thus this cofactor has a DSD with $a$ separated.

Only if. Suppose all the $b$ cofactors have DSDs with variables $a$ in separate blocks. Thus $F_{b^{j}}(a, c)=H_{j}\left(D_{j}(a), c\right)$ for some functions $H_{j}$ and $D_{j}$. We can take $D_{j}(a)=0$ if $b^{j}$ is $\mathrm{bsi}^{2}$. The Shannon expansion gives $F(a, b, c)=\sum_{j=0}^{2^{|b|}-1} b^{j} H_{j}\left(D_{j}(a), c\right)$. Define $D(a, b)=\sum_{j=0}^{2^{\mid b}-1} b^{j} D_{j}(a)$ and note that $b^{j} H_{j}\left(D_{j}(a), c\right)=b^{j} H_{j}\left(\sum_{m=0}^{2^{|b|}-1} b^{m} D_{m}(a), c\right)$. Thus, $F(a, b, c)=\sum_{j=0}^{2^{|b|}-1} b^{j} H_{j}\left(\sum_{m=0}^{2^{|b|}-1} b^{m} D_{m}(a), c\right)=H(D(a, b), b, c)$. QED.

In many applications, e.g. Section 4 , the shared variables $b$ are selected first, and the bound set variables $a$ are found using Theorem 3.1 to search for a largest set $a$ that can be used (thus obtaining the maximum support reduction).
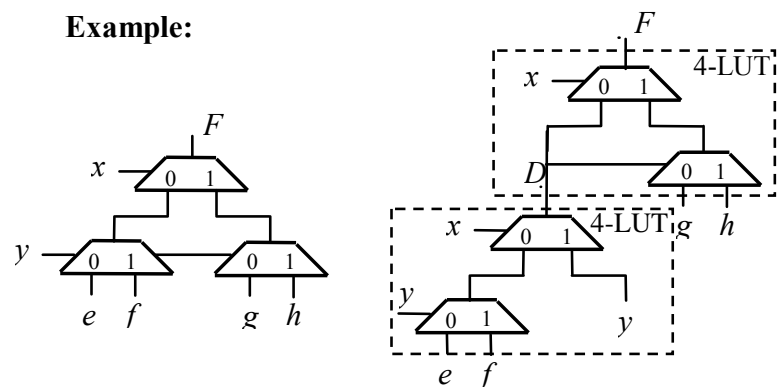

Figure 3.1. Mapping 4:1 MUX into two 4-LUTs.

Consider the problem of decomposing a 4:1 MUX into two 4-LUTs. A structural mapper starting from the structure shown in Figure 3.1 on the left would require three 4-LUTs, each containing a 2:1 MUX. To achieve a more compact mapping, we find a decomposition with respect to $(a, b)=((e, f, y), x)$. The free variables are $c=(g, h)$. This leads to cofactors $F_{\bar{x}}=\bar{y} e+y f$ and $F_{x}=\bar{y} g+y h$. Both $F_{\bar{x}}$ and $F_{x}$ have $(e, f, y)$

\footnotetext{
${ }^{2}$ The choice of taking $D_{j}(a)=0$ is arbitrary. We could have equally well
} taken $D_{j}(a)$ to be any function of $a$ 
as a separate block ${ }^{3}$. Thus, $D_{0}=\bar{y} e+y f$ and $D_{1}=y$, while $H_{0}=D_{0}$ and $H_{1}=\bar{D}_{1} g+D_{1} h$. Thus we can write $F=\bar{x} H_{0}+x H_{1}=\bar{x}\left(D_{0}\right)+x\left(\bar{D}_{1} g+D_{1} h\right)$. Replacing $D_{0}$ and $D_{1} \quad$ with $\quad D=\bar{x}(\bar{y} e+y f)+x(y)$, we have $F=\bar{x} D+x(\bar{D} g+D h)$. This leads to the decomposition shown on the right of Figure 3.1. As a result, a 4:1 MUX is realized by two 4-LUTs.

We will use the notation $f \cong g$ to denote that $f$ equals $g$ up to complementation. The following theorem characterizes the set of all $(a, b)$-divisors of a function $F$.

Theorem 3.2: Let $F$ have an $(a, b)$-decomposition with an associated divisor $\quad D(a, b)=\sum_{j=0}^{2^{|b|}-1} b^{j} D_{j}(a)$. Then $\hat{D}(a, b)=\sum_{j=0}^{2^{|b|}-1} b^{j} \widehat{D}_{j}(a)$ is also an $(a, b)$-divisor if and only if $\widehat{D}_{j}(a) \cong D_{j}(a), \forall j \in J$, where $J$ is the set of indices of the bsdcofactors of $F$.

Proof: If. Suppose $\quad \widehat{D}_{j}(a) \cong D_{j}(a), \forall j \in J$. If $\hat{D}(a, b)=\sum_{j=0}^{2^{|b|}-1} b^{j} \hat{D}_{j}(a) \quad$ where $\quad \hat{D}_{j}(a), j \notin J \quad$ is an arbitrary function, we have to show that $\widehat{D}(a, b)$ is a divisor of $F(a, b, c)$. We are given that $D(a, b)=\sum_{j=0}^{2^{|b|}-1} b^{j} D_{j}(a)$ is a divisor of $F(a, b, c)$. Thus, there exists $H$, such that $F=H(D(a, b), b, c)=$ $\sum_{j=0}^{2^{|b|}-1} b^{j} H_{b^{j}}\left(D_{j}(a), c\right)=\sum_{j \in J_{1} \cup J_{2}} b^{j} H_{b^{j}}\left(D_{j}(a), c\right)+\sum_{j \notin J} b^{j} H_{b^{j}}\left(D_{j}(a), c\right)$ where $J_{1}=\left\{j \mid \hat{D}_{j}(a)=D_{j}(a)\right\} \quad$ and $\quad J_{2}=\left\{j \mid \hat{D}_{j}(a)=\bar{D}_{j}(a)\right\}$. Clearly $J=J_{1} \cup J_{2}$. Now define the operator $H_{k}^{\prime}(\bullet, c)=H_{k}(\bar{\bullet}, c)$, i.e. it takes a function and inverts its first argument. Thus, $H_{j}\left(D_{j}(a), c\right)=H_{j}\left(\widehat{D}_{j}(a), c\right), j \in J_{1} \quad$ and $H_{j}\left(D_{j}(a), c\right)=H_{j}^{\prime}\left(\widehat{D}_{j}(a), c\right), j \in J_{2}$. Finally, $H_{j}\left(D_{j}(a), c\right)=H_{j}\left(\hat{D}_{j}(a), c\right), j \notin J$, since $H_{j}$ does not depend on the first variable. Thus

$F=\sum_{j \in J_{1}} b^{j} H_{b^{j}}\left(\hat{D}_{j}(a), c\right)+\sum_{j \in J_{2}} b^{j} H_{b^{j}}^{\prime}\left(\hat{D}_{j}(a), c\right)+\sum_{j \notin J} b^{j} H_{b^{j}}\left(\hat{D}_{j}(a), c\right)$.

Thus $F=\hat{H}(\hat{D}(a, b), b, c)$, where

$\widehat{H} \equiv \sum_{j \in J_{1}} b^{j} H_{b^{j}}(\bullet, c)+\sum_{j \in J_{2}} b^{j} H_{b^{j}}^{\prime}(\bullet, c)+\sum_{j \notin J} b^{j} H_{b^{j}}(\bullet, c)$.

Therefore $\hat{D}(a, b)$ is an $(a, b)$-divisor of $F$.

Only if. Assume that $F=H(D(a, b), b, c)$ and $F=\hat{H}(\hat{D}(a, b), b, c)$. Cofactoring each with respect to $b^{j}, j \in J$,

\footnotetext{
${ }^{3}$ In $F_{x}$, the DSD is a trivial one in which each input is a separate block. The variables $(e, f)$ do not appear in $F_{x}$, but are considered as part of the separate block containing $y$. Similarly, in $F_{\bar{x}}$ the entire cofactor is a separate block.
}

yields $\quad F_{b^{j}}=H_{b^{j}}\left(D_{b^{j}}(a), c\right)$ and $F_{b^{j}}=\hat{H}_{b^{j}}\left(\hat{D}_{b^{j}}(a), c\right)$. Thus $F_{b^{j}}(a, c)$ has a DSD with respect to $a$, and by Theorem 2.1, $D_{b^{j}}(a) \cong \widehat{D}_{b^{j}}(a)$ for $b^{j}, j \in J$. QED

Note that even up to complementation, divisors are not unique since those associated with bsi cofactor literals can be chosen as arbitrary functions of $a$.

Example: $F=a b+\bar{b} c=(a b+\bar{a} \bar{b}) b+\bar{b} c$ The bsd-cofactors are $\{b\}$ and the bsi-cofactors are $(\bar{b}\}$ since $F_{\bar{b}}=c$ is independent of $a . F$ has $(a, b)$-divisors $D^{1}=a b$ and $D^{2}=(a b+\bar{a} \bar{b})$, which agree in the bsd-cofactor $b$, i.e. $D_{b}^{1}(a)=D_{b}^{2}(a)$. In addition, $D^{3}=\overline{D^{1}}=\bar{a}+\bar{b}$ is a divisor because $F=\overline{D^{3}}+\bar{b} c$.

In contrast to the discussion so far, the next result deals with finding common Boolean divisors among a set of functions.

Definition: Two functions, $F_{1}, F_{2}$, are said to be $(a, b)$ compatible if each has an $(a, b)$-divisor, and $\forall j \in J_{1} \cap J_{2}$, $D_{b^{j}}^{1}(a) \cong D_{b^{j}}^{2}(a)$, where $J_{i}$ is the set of $b s d b$-cofactors of $F_{i}$.

We emphasize that the cofactor divisors of the two functions need only match for the bound set dependent (bsd) cofactors.

Theorem 3.3 $\mathbf{4}^{\mathbf{4}}$ : There exists a common $(a, b)$-divisor of $\left\{F_{1}, \cdots, F_{n}\right\}$ if and only if the set $\left\{F_{1}, \cdots, F_{n}\right\}$ is pair-wise $(a, b)$ compatible.

Proof: For simplicity, we show the proof for $n=2$ and note that pairwise compatibility is required because compatibility is not transitive. The common divisor will have a uniquely specified cofactor (up to complementation) only if at least one of the functions has this cofactor as bsd.

If. Suppose $F_{1}$ and $F_{2}$ are $(a, b)$-compatible. Then $F_{1}(a, b, c)=H_{1}\left(D^{1}(a, b), b, c\right)$ and $F_{2}(a, b, c)=H_{2}\left(D^{2}(a, b), b, c\right)$ and $D_{b^{j}}^{1}(a) \cong D_{b^{j}}^{2}(a)$ for all bsd $\left\{b^{j}\right\}$ for both $F_{1}$ and $F_{2}$. Define $\tilde{D}_{b^{j}}(a)=D_{b^{j}}^{1}(a)$ for such $b^{j}$. If $b^{j}$ is bsd for $F_{1}$ and bsi for $F_{2}$, let $\tilde{D}_{b^{j}}(a)=D_{b^{j}}^{1}(a)$. If $b^{j}$ is bsd for $F_{2}$ and bsi for $F_{1}$ let $\tilde{D}_{b^{j}}(a)=D_{b^{j}}^{2}(a)$. Otherwise, let $\tilde{D}_{b^{j}}(a)=0$. Clearly, by Theorem 3.2, $\tilde{D}(a, b)=\sum_{j=0}^{2^{|b|}-1} b^{j} \tilde{D}_{b^{j}}(a)$ is an $(a, b)$-divisor of both $F_{1}$ and $F_{1}$.

Only if. Suppose a common $(a, b)$-divisor exists, i.e. $F_{1}(a, b, c)=H_{1}(\tilde{D}(a, b), b, c) \quad$ and $\quad F_{2}(a, b, c)=H_{2}(\tilde{D}(a, b), b, c)$. Then both $F_{1}$ and $F_{2}$ have $(a, b)$-divisors such that $D_{b^{j}}^{1}(a) \cong D_{b^{j}}^{2}(a)$ for $j \in J_{1} \cap J_{2}$, namely, $D^{1}=D^{2}=\tilde{D}$. QED

Thus a common divisor of two functions with shared variable $b$ can be found by i) cofactoring with respect to $b$, ii) computing the maximum DSDs of the cofactors, and iii) looking for a set of variables $a$ for which the associated cofactors are compatible.

\footnotetext{
${ }^{4}$ As far as we know, there is no equivalent theorem in the literature.
} 


\section{Rewriting $K$-LUT networks}

We consider a new method for rewriting $K$-LUT networks, using the ideas of Section 3, and discuss a particular implementation with experimental results.

\subsection{Global view}

The objective is to rewrite a local window of a $K$-LUT mapped network. The window consists of a root LUT, $n$, and a certain number of transitive fanin (TFI) LUTs. The TFI LUTs are associated with a cut $C$. The local network to be rewritten consists of the LUT for node $n$ plus all LUTs between $C$ and $n$. Our objective is to re-decompose the associated function of $n, f_{n}(C)$, expressed using the cut variables, into a smaller number of LUTs. For convenience, we denote this local network of LUTs as $N_{n}$.

The maximum fanout free cone (MFFC) of $N_{n}$ is the set of LUTs in $N_{n}$, which fanout to nodes only in the fanin cone of $n$. Thus if node $n$ were removed, all nodes in $\operatorname{MFFC}(n)$ could be removed also. We want to re-decompose $N_{n}$ into fewer $K$-LUTs taking into account that shared LUTs, not in $\operatorname{MFFC}(n)$, must remain. Since it is unlikely that an improvement will be found when a cut has a small $\operatorname{MFFC}(n)$, we only consider cuts with no more than $S$ shared LUTs (in our experiments we set $S=3$ ).

Given $n$ and a cut $C(n)$, the problem is to find a decomposition of $f_{n}(C)$ composed of the minimum number of $K$ (or less) input blocks. For those $N_{n}$ where there is a gain (accounting for duplication of the LUTs not in the MFFC), we replace $N_{n}$ with its new decomposition.

The efficacy of this method depends on the following:

- the order in which the nodes $n$ are visited,

- the cut selected for rewriting $f_{n}(C)$,

- not using decompositions that worsen delay,

- creating a more balanced decomposition, and

- pre-processing to detect easy decompositions ${ }^{5}$.

We describe the most important aspect of this problem, which is finding a maximum support-reducing decomposition of a function $F$. Other details of the algorithm can be found in [32] and in the source code of $\mathrm{ABC}$ [5] implementing command lutpack.

The proposed algorithm works by cofactoring, with respect to different sets $b$, the non-decomposable blocks of the DSD of $F$ and using Theorem 3.1 to find a Boolean divisor and bound variables $a$. The approach greedily extracts a maximum supportreducing block at each step.

Although any optimum implementation of a $K$-LUT network must be support reducing if any fanin of a block is support reducing for that block, in general, it may not be maximum support-reducing.

\subsection{Finding the maximum support-reducing decomposition}

The approach is to search for common bound-sets of the cofactor DSDs where cofactoring is tried with respect to subsets of variables in the support of $F$. If all subsets are attempted, the proposed greedy approach reduces the associated block to a minimum number of inputs. We trade-off the quality of the decomposition found for runtime spent in exploring cofactoring sets. A limit is imposed on (a) the number of cofactoring variables, and (b) the number of variable combinations tried.

\footnotetext{
${ }^{5}$ e.g. a MUX decomposition of a function with at most $2 K-1$ inputs with cofactors of input size $K-2$ and $K$.
}

Experimentally, we found that this heuristic usually finds a solution with a minimum number of blocks.

The pseudo-code in Figure 4.1 shows how the DSD structures of the cofactors can be exploited to compute a bound set that leads to the maximum support reduction during constructive decomposition. findSupportReducingBoundSet takes a completely-specified function $F$ and the limit $K$ on the support size of the decomposed block. It returns a bound-set leading to the decomposition with a maximal support-reduction. It returns NONE if a support-reducing decomposition does not exist.

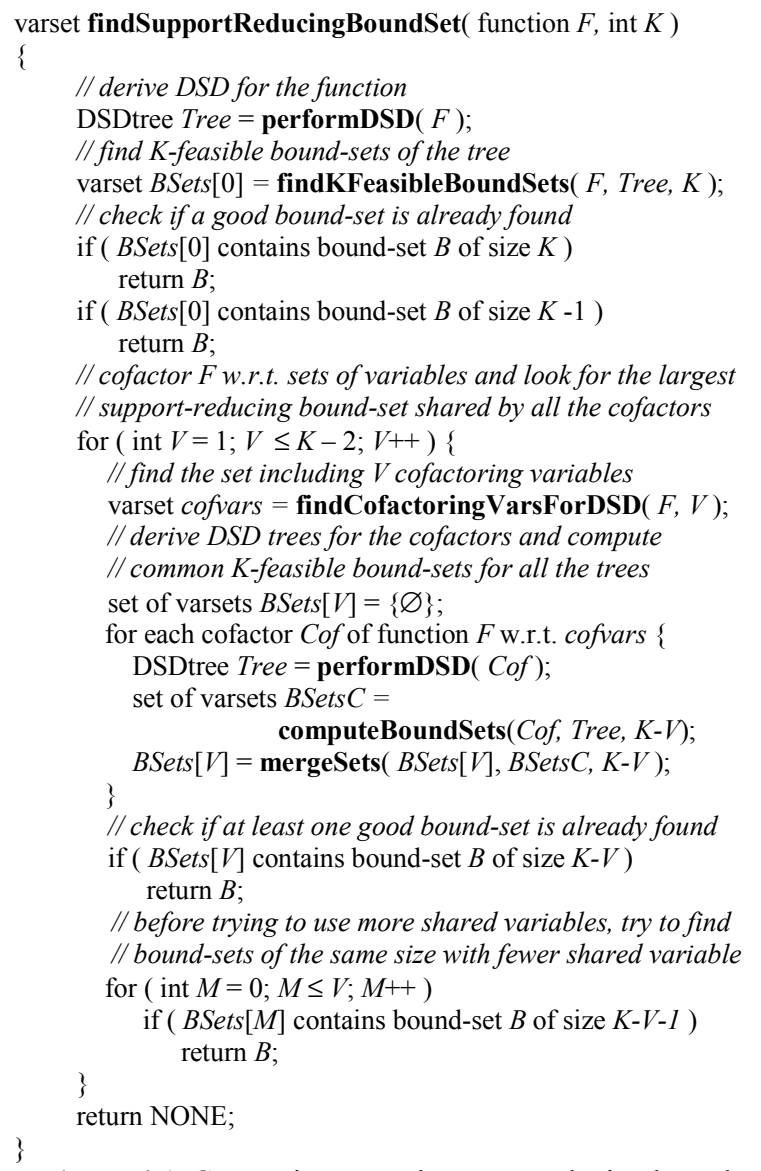

Figure 4.1. Computing a good support-reducing bound-set.

The procedure first derives the maximum DSD tree of the function itself. The tree is used to compute the set of all feasible bound-sets whose size is $K$ or less. Larger sets are of no interest because they cannot be implemented using $K$-LUTs. For such bound-sets, decomposition with a single output and no shared variables is possible, and if one is found, it is returned. If no such bound set exists (for example, when the function has no DSD), the computation enters a loop, in which cofactoring is tried, and common support-reducing bound-sets of the cofactors are explored. Cofactoring with respect to one variable is tried first. If the two cofactors of the function have DSDs with a common bound-set of size $K-1$, it is returned. In this case, although the divisor block has $K$ variables, the support of $F$ is only reduced by $K-2$ because the cofactoring variable is shared and the output of the block is a new input. If there is no common bound-set of size $K-1$, the next best outcome is one of the following:

1. There is a bound-set of size $K-2$ of the original function.

2. There is a common bound-set of size $K-2$ of the two cofactors with respect to the cofactoring variable. 
3. There is a common bound-set of size $K-2$ of the four cofactors with respect to two variables.

The loop over $M$ at the bottom of Figure 4.1 tests for outcomes (1) and (2). If these are impossible, $V$ is incremented and the next iteration of the loop is performed, which is the test for the outcome (3).

In the next iteration over $V$, cofactoring with respect to two variables is attempted and the four resulting cofactors are searched for common bound-sets. The process is continued until a bound-set is found, or cofactoring with $K-2$ variables is tried without success. When $V=K-1$, the decomposition is not supportreducing, because the dividend function depends on $K-1$ shared variables plus the output of the decomposed block. In this case, NONE is returned.

Example: Consider the decomposition of function $F$ of the 4:1 MUX shown in Figure 3.1 (left). Assume $K=4$. This function has a maximum DSD composed of one prime block. The set of $K$-feasible bound-sets is just $\{\{\varnothing\},\{a\},\{b\},\{c\},\{d\},\{x\},\{y\}\}$. The above procedure enters the loop with $V=1$. Suppose $x$ is chosen as the cofactoring variable. The cofactors are $F_{\bar{x}}=\bar{y} a+y b$ and $F_{x}=\bar{y} c+y d$. The $K-1-$ feasible bound-sets are $\{\{\varnothing\},\{a\},\{b\},\{y\},\{a, b, y\}\}$, and $\{\{\varnothing\},\{c\},\{d\},\{y\},\{c, d, y\}\}$. A common bound-set $\{a, b, y\}$ of size $K-1$ exists. The loop terminates and this bound-set is returned, resulting in the decomposition in Figure 3.1 (right).

\subsection{Experimental results}

The proposed algorithm is implemented in $\mathrm{ABC}$ [5] as command lutpack. Experiments targeting 6-input LUTs were run on an Intel Xeon 2-CPU 4-core computer with 8GB of RAM. The resulting networks were all verified using the combinational equivalence checker in $\mathrm{ABC}$ (command cec) [28].

The following $\mathrm{ABC}$ commands are included in the scripts used in the experiments, which targeted area minimization while preserving delay:

- resyn is a logic synthesis script that runs 5 iterations of AIG rewriting [27] to improve area without increasing depth;

- resyn 2 is a script that performs 10 iterations of a more diverse set of AIG rewritings than those of resyn;

- choice is a script that allows for accumulation of structural choices; choice runs resyn followed by resyn 2 and collects three snapshots of the network: the original, the final, and the one after resyn, resulting in a circuit with structural choices;

- if is an efficient FPGA mapper using priority cuts [31], finetuned for area recovery (after a minimum delay mapping) and using subject graphs with structural choices ${ }^{6}$;

- $i m f s$ is an area-oriented resynthesis engine for FPGAs [30] based on changing a logic function at a node by extracting don't cares from a window and using Boolean resubstitution to rewrite the node function using possibly new inputs; and

- lutpack is the new resynthesis described in this section.

The benchmarks used are 20 large public benchmarks from the MCNC and ISCAS'89 suites found in previous work on FPGA mapping [22][11][29] $]^{7}$.

Table 1 shows four experimental runs. We use an exponent notation to denote iteration of the expression in parenthesis $n$ times, e.g. $(\mathrm{com} 1 ; \operatorname{com} 2)^{3}$ means iterate (com 1; com 2$)$ three times.

\footnotetext{
${ }^{6}$ The mapper was run with the following settings: at most 12 6-input priority cuts are stored at each node; five iterations of area recovery are performed, three with area flow and two with exact local area.

${ }^{7}$ In the above set, circuit s298 was replaced by i10 because the former contains only 24 6-LUTs
}

- "Baseline" = $($ resyn $;$ resyn $2 ;$ if $)$. It corresponds to a typical run of technology-independent synthesis followed by default mapping into 6-LUTs.

- "Choices" = resyn; resyn 2; if; (choice; if $)^{4}$.

- "Imfs" = resyn; resyn 2; if; (choice; if; imfs $)^{4}$

- "Lutpack" = resyn; resyn2; if; (choice; if; imfs $)^{4}$; $(\text { lutpack })^{2}$. The table lists the number of primary inputs ("PIs"), primary outputs ("POs"), registers ("Reg"), area calculated as the number of 6-LUTs ("LUT") and delay calculated as the depth of the 6-LUT network ("Level"). The ratios in the tables are the ratios of the geometric averages of values reported in the columns.

The main purpose of the experiments was to demonstrate the additional impact that the proposed command lutpack has on top of a strong synthesis flow. The Baseline, Choices and Imfs columns show that repeated re-mapping and use of don't cares in a window already has a dramatic impact over strong conventional mapping (Baseline). The last line of the table compares lutpack against the strongest result obtained using other methods ( $\mathrm{imfs}$ ). Given the power of $i m f s$, it is somewhat unexpected that lutpack can achieve an additional 5.4\% reduction in area.

This additional area reduction indicates the orthogonal nature of lutpack and imfs. While $i m f s$ tries to reduce area by analyzing alternative resubstitutions at each node (using don't cares), it cannot efficiently compact large fanout-free cones that may be present in the mapping. The latter is done by lutpack, which iteratively re-factors fanout-free cones (up to 16 inputs) and finds new implementations using a minimal number of LUTs.

Runtimes are not included in Table 1 but, as an indication, one run of lutpack did not exceed $20 \mathrm{sec}$ for any of the benchmarks. The total runtime of the experiments was dominated by imfs. Table 1 illustrates only two passes of lutpack as a final processing, but experiments where lutpack is in the iteration loop, e.g. (choice; if; imfs; lutpack) ${ }^{4}$, often show similar additional gains.

\section{Conclusions and future work}

The paper presented a fast algorithm for decomposition of logic functions. It was applied to area-oriented resynthesis of $K$-LUT structures and implemented in $\mathrm{ABC}$ as command lutpack, which is based on cofactoring and disjoint-support decomposition. The computation is faster than previous solutions relying on BDDbased decomposition and Boolean satisfiability. It achieved an additional $5.4 \%$ reduction in area, when applied to networks obtained by iterated high-quality technology mapping and powerful resynthesis using don't cares and windowing.

Future work will include:

- Improving the DSD-based analysis, which occasionally fails to find a feasible match and is the most time-consuming part.

- Exploring other data structures for cofactoring and DSD decomposition, to allow processing of functions with more than 16 inputs. This will improve the quality of resynthesis.

Some possible future applications include:

1. computing all decompositions of a function and using them to find common Boolean divisors among all functions of a logic network,

2. merging fanin blocks of two functions, where the blocks share common supports,

3. extracting a common Boolean divisor from a pair of functions (using Theorem 3.3),

4. mapping into fixed macro-cells, where the divisors must have a fixed given structure as exemplified by Altera Stratix II [3] or Actel ProASIC3 devices [2]. 


\section{Acknowledgements}

This work was supported in part by SRC contracts 1361.001 and 1444.001, NSF contract CCF-0702668, and the California MICRO Program with industrial sponsors Actel, Altera, Calypto, IBM, Intel, Intrinsity, Magma, Synopsys, Synplicity, Tabula, and Xilinx. Thanks to Stephen Jang of Xilinx for his masterful experimental evaluation of the proposed algorithms, to Slawomir Pilarski and Victor Kravets for careful readings and useful comments, and to an anonymous reviewer for pointing out critical errors in the original manuscript. This work was done while the third author was at Berkeley.

\section{References}

[1] A. Abdollahi and M. Pedram, "A new canonical form for fast Boolean matching in logic synthesis and verification", Proc. DAC '05, pp. 379-384.

[2] Actel Corp., "ProASIC3 flash family FPGAs datasheet," http://www.actel.com/documents/PA3 DS.pdf

[3] Altera Corp., "Stratix II device family data sheet", 2005, http://www.altera.com/literature/hb/stx/stratix_section_1_vol_1.pdf

[4] R. L. Ashenhurst, "The decomposition of switching functions", Proc. Intl Symposium on the Theory of Switching, Part I (Annals of the Computation Laboratory of Harvard University, Vol. XXIX), Harvard University Press, Cambridge, 1959, pp. 75-116.

[5] Berkeley Logic Synthesis and Verification Group, ABC: A System for Sequential Synthesis and Verification, Release 70911. http://www.eecs.berkeley.edu/ alanmi/abc/

[6] V. Bertacco and M. Damiani. "The disjunctive decomposition of logic functions". Proc. ICCAD '97, pp. 78-82.

[7] R. Brayton and C. McMullen, "The decomposition and factorization of Boolean expressions," Proc. ISCAS '82, pp. 29-54.

[8] D. Chai and A. Kuehlmann, "Building a better Boolean matcher and symmetry detector,” Proc. DATE ‘06, pp. 1079-1084.

[9] S. Chatterjee, A. Mishchenko, and R. Brayton, "Factor cuts", Proc. ICCAD '06, pp. 143-150. http://www.eecs.berkeley.edu/ alanmi/ publications/2006/iccad06_cut.pdf

[10] S. Chatterjee, A. Mishchenko, R. Brayton, X. Wang, and T. Kam, "Reducing structural bias in technology mapping", Proc. ICCAD '05, pp. 519-526. http://www.eecs.berkeley.edu/ alanmi/ publications/2005/iccad05_map.pdf

[11] D. Chen and J. Cong. "DAOmap: A depth-optimal area optimization mapping algorithm for FPGA designs," Proc. ICCAD '04, 752-757.

[12] J. Cong, C. Wu, and Y. Ding, "Cut ranking and pruning: Enabling a general and efficient FPGA mapping solution," Proc. FPGA'99, pp. 29-36.

[13] A. Curtis. New approach to the design of switching circuits. Van Nostrand, Princeton, NJ, 1962.

[14] D. Debnath and T. Sasao, "Efficient computation of canonical form for Boolean matching in large libraries," Proc. ASP-DAC '04, pp. 591-596.

[15] A. Farrahi and M. Sarrafzadeh, "Complexity of lookup-table minimization problem for FPGA technology mapping," IEEE TCAD, Vol. 13(11), Nov. 1994, pp. 1319-1332.

[16] C. Files and M. Perkowski, "New multi-valued functional decomposition algorithms based on MDDs". IEEE TCAD, Vol. 19(9) , Sept. 2000, pp. 1081-1086.

[17] Y. Hu, V. Shih, R. Majumdar, and L. He, "Exploiting symmetry in SAT-based Boolean matching for heterogeneous FPGA technology mapping", Proc. ICCAD '07.

[18] V. N. Kravets. Constructive Multi-Level Synthesis by Way of Functional Properties. Ph. D. Thesis. University of Michigan, 2001.

[19] V. N. Kravets and K. A. Sakallah, "Constructive library-aware synthesis using symmetries", Proc. of DATE, pp. 208-213, March 2000 .
[20] E. Lehman, Y. Watanabe, J. Grodstein, and H. Harkness, "Logic decomposition during technology mapping," IEEE TCAD, Vol. 16(8), 1997, pp. 813-833.

[21] A. Ling, D. Singh, and S. Brown, "FPGA technology mapping: A study of optimality", Proc. DAC '05, pp. 427-432.

[22] V. Manohara-rajah, S. D. Brown, and Z. G. Vranesic, "Heuristics for area minimization in LUT-based FPGA technology mapping," Proc. IWLS '04, pp. 14-21.

[23] Y. Matsunaga. "An exact and efficient algorithm for disjunctive decomposition". Proc. SASIMI '98, pp. 44-50.

[24] K. Minkovich and J. Cong, "An improved SAT-based Boolean matching using implicants for LUT-based FPGAs," Proc. FPGA'07.

[25] A. Mishchenko and T. Sasao, "Encoding of Boolean functions and its application to LUT cascade synthesis", Proc. IWLS '02, pp. 115120. http://www.eecs.berkeley.edu/ alanmi/publications/2002/ iwls02_enc.pdf

[26] A. Mishchenko, X. Wang, and T. Kam, "A new enhanced constructive decomposition and mapping algorithm", Proc. DAC '03, pp. 143-148.

[27] A. Mishchenko, S. Chatterjee, and R. Brayton, "DAG-aware AIG rewriting: A fresh look at combinational logic synthesis", Proc. $D A C^{\prime} 06$, pp. 532-536.

[28] A. Mishchenko, S. Chatterjee, R. Brayton, and N. Een, "Improvements to combinational equivalence checking", Proc. ICCAD '06, pp. 836-843. http://www.eecs.berkeley.edu/ $\sim$ alanmi/publications/2006/iccad06_cec.pdf

[29] A. Mishchenko, S. Chatterjee, and R. Brayton, "Improvements to technology mapping for LUT-based FPGAs". IEEE TCAD, Vol. 26(2), Feb 2007, pp. 240-253. http:/www.eecs.berkeley.edu/ alanmi/publications/2006/tcad06_map.pdf

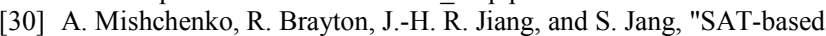
logic optimization and resynthesis". Submitted to FPGA'08. http://www.eecs.berkeley.edu/ alanmi/publications/2008/ fpga08_imfs.pdf

[31] A. Mishchenko, S. Cho, S. Chatterjee, and R. Brayton, "Combinational and sequential mapping with priority cuts", Proc. ICCAD '07. http://www.eecs.berkeley.edu/ alanmi/ publications/2007/iccad07_map.pdf

[32] A. Mishchenko, S. Chatterjee, and R. Brayton, "Fast Boolean matching for LUT structures". ERL Technical Report, EECS Dept., UC Berkeley. http://www.eecs.berkeley.edu/ alanmi/publications/ 2007/tech07 lpk.pdf

[33] P. Pan and C.-C. Lin, "A new retiming-based technology mapping algorithm for LUT-based FPGAs," Proc. FPGA '98, pp. 35-42.

[34] M. Perkowski, M. Marek-Sadowska, L. Jozwiak, T. Luba, S. Grygiel, M. Nowicka, R. Malvi, Z. Wang, and J. S. Zhang. "Decomposition of multiple-valued relations". Proc. ISMVL'97, pp. 13-18.

[35] S. Plaza and V. Bertacco, "Boolean operations on decomposed functions", Proc. IWLS' 05, pp. 310-317.

[36] J. P. Roth and R. Karp, "Minimization over Boolean graphs", IBM J. Res. and Develop., 6(2), pp. 227-238, April 1962.

[37] S. Safarpour, A. Veneris, G. Baeckler, and R. Yuan, "Efficient SATbased Boolean matching for FPGA technology mapping," Proc. $D A C ' 06$.

[38] H. Sawada, T. Suyama, and A. Nagoya, "Logic synthesis for lookup tables based FPGAs using functional decomposition and support minimization", Proc. ICCAD, 353-358, Nov. 1995.

[39] T. Sasao and M. Matsuura, "DECOMPOS: An integrated system for functional decomposition," Proc. IWLS '98, pp. 471-477.

[40] N. Vemuri and P. Kalla and R. Tessier, "BDD-based logic synthesis for LUT-based FPGAs", ACM TODAES, Vol. 7, 2002, pp. 501-525.

[41] B. Wurth, U. Schlichtmann, K. Eckl, and K. Antreich. "Functional multiple-output decomposition with application to technology mapping for lookup table-based FPGAs". ACM Trans. Design Autom. Electr. Syst. Vol. 4(3), 1999, pp. 313-350. 
Table 1. Evaluation of resynthesis applied after technology mapping for FPGAs $(K=6)$.

\begin{tabular}{|c|c|c|c|c|c|c|c|c|c|c|c|}
\hline \multirow{2}{*}{ Designs } & \multirow{2}{*}{ PI } & \multirow{2}{*}{$\mathrm{PO}$} & \multirow{2}{*}{ Reg } & \multicolumn{2}{|c|}{ Baseline } & \multicolumn{2}{|c|}{ Choices } & \multicolumn{2}{|c|}{ Imfs } & \multicolumn{2}{|c|}{ Imfs + Lutpack } \\
\hline & & & & LUT & Level & LUT & Level & LUT & Level & LUT & Level \\
\hline alu4 & 14 & 8 & 0 & 821 & 6 & 785 & 5 & 558 & 5 & 453 & 5 \\
\hline apex2 & 39 & 3 & 0 & 992 & 6 & 866 & 6 & 806 & 6 & 787 & 6 \\
\hline apex4 & 9 & 19 & 0 & 838 & 5 & 853 & 5 & 800 & 5 & 732 & 5 \\
\hline bigkey & 263 & 197 & 224 & 575 & 3 & 575 & 3 & 575 & 3 & 575 & 3 \\
\hline clma & 383 & 82 & 33 & 3323 & 10 & 2715 & 9 & 1277 & 8 & 1222 & 8 \\
\hline des & 256 & 245 & 0 & 794 & 5 & 512 & 5 & 483 & 4 & 480 & 4 \\
\hline diffeq & 64 & 39 & 377 & 659 & 7 & 632 & 7 & 636 & 7 & 634 & 7 \\
\hline dsip & 229 & 197 & 224 & 687 & 3 & 685 & 2 & 685 & 2 & 685 & 2 \\
\hline $\operatorname{ex1010}$ & 10 & 10 & 0 & 2847 & 6 & 2967 & 6 & 1282 & 5 & 1059 & 5 \\
\hline ex5p & 8 & 63 & 0 & 599 & 5 & 669 & 4 & 118 & 3 & 108 & 3 \\
\hline elliptic & 131 & 114 & 1122 & 1773 & 10 & 1824 & 9 & 1820 & 9 & 1819 & 9 \\
\hline frisc & 20 & 116 & 886 & 1748 & 13 & 1671 & 12 & 1692 & 12 & 1683 & 12 \\
\hline $\mathrm{i} 10$ & 257 & 224 & 0 & 589 & 9 & 560 & 8 & 548 & 7 & 547 & 7 \\
\hline pdc & 16 & 40 & 0 & 2327 & 7 & 2500 & 6 & 194 & 5 & 171 & 5 \\
\hline misex3 & 14 & 14 & 0 & 785 & 5 & 664 & 5 & 517 & 5 & 446 & 5 \\
\hline s38417 & 28 & 106 & 1636 & 2684 & 6 & 2674 & 6 & 2621 & 6 & 2592 & 6 \\
\hline s38584 & 12 & 278 & 1452 & 2697 & 7 & 2647 & 6 & 2620 & 6 & 2601 & 6 \\
\hline seq & 41 & 35 & 0 & 931 & 5 & 756 & 5 & 682 & 5 & 645 & 5 \\
\hline spla & 16 & 46 & 0 & 1913 & 6 & 1828 & 6 & 289 & 4 & 263 & 4 \\
\hline tseng & 52 & 122 & 385 & 647 & 7 & 649 & 6 & 645 & 6 & 645 & 6 \\
\hline geomean & & & & 1168 & 6.16 & 1103 & 5.66 & 716 & 5.24 & 677 & 5.24 \\
\hline Ratio1 & & & & 1.000 & 1.000 & 0.945 & 0.919 & 0.613 & 0.852 & 0.580 & 0.852 \\
\hline Ratio2 & & & & & & 1.000 & 1.000 & 0.649 & 0.926 & 0.614 & 0.926 \\
\hline Ratio3 & & & & & & & & 1.000 & 1.000 & 0.946 & 1.000 \\
\hline
\end{tabular}

